\title{
Probing Features of the Lee-Wick Quantum Electrodynamics
}

\author{
R. Turcati ${ }^{1,2}$ and M. J. Neves ${ }^{3}$ \\ ${ }^{1}$ Departamento de Física e Química, Universidade Federal do Espírito Santo, Avenida Fernando Ferrari 514, \\ Goiabeiras, 29060-900 Vitória, ES, Brazil \\ ${ }^{2}$ Laboratório de Fsica Experimental (LAFEX), Centro Brasileiro de Pesquisas Físicas (CBPF), Rua Dr. Xavier Sigaud 150, \\ Urca, 22290-180 Rio de Janeiro, RJ, Brazil \\ ${ }^{3}$ Departamento de Física, Universidade Federal Rural do Rio de Janeiro, BR 465-07, 23890-971 Seropédica, RJ, Brazil
}

Correspondence should be addressed to R. Turcati; rturcati@gmail.com

Received 2 June 2014; Revised 5 September 2014; Accepted 14 September 2014; Published 13 October 2014

Academic Editor: Alexey A. Petrov

Copyright (C) 2014 R. Turcati and M. J. Neves. This is an open access article distributed under the Creative Commons Attribution License, which permits unrestricted use, distribution, and reproduction in any medium, provided the original work is properly cited. The publication of this article was funded by SCOAP . $^{3}$

\begin{abstract}
We discuss some aspects concerning the electromagnetic sector of the abelian Lee-Wick (LW) quantum electrodynamics (QED). Using the Dirac's theory of constrained systems, the higher-order canonical quantization of the LW electromagnetism is performed. A quantum bound on the LW heavy mass is also estimated using the best known measurement of the anomalous magnetic moment of the electron. Finally, it is shown that magnetic monopoles can coexist peacefully in the LW scenario.
\end{abstract}

\section{Introduction}

In recent years, B. Grinstein, D. O'Connell, and M. Wise extended the ideas of the LW finite QED $[1,2]$ to nonabelian gauge theories and gave rise to the Lee-Wick standard model (LWSM) [3]. In the LWSM, each field of the standard model (SM) has associated a massive LW partner, with these modes being the only parameters added to the SM framework. LW theories belong to a class of higher-order gauge models that are very useful to treat ultraviolet divergences. By adding higher-order derivative kinetic terms in the Lagrangian density, the modified propagator improves the behavior at high energies scale and induces the appearance of gauge massive resonances. Unlike the original LW QED, the LWSM is not finite, but it is renormalizable. The reason is because the gauge covariant derivate introduces momentum dependence interactions, giving rise to degree of divergence of loops diagrams and the modified propagators have better asymptotic behavior in the ultraviolet range, reducing the degree of divergence in radiative corrections. By power counting arguments, these mutual effects cancel each other and give origin at most to logarithmic divergences, providing an alternative way to solve the hierarchy puzzle [3]. It also have been suggested that quantum gravity effects can excite a
Lee-Wick partner to every field in the SM $[4,5]$, which is the exact degree of freedom required by the LWSM. In order to be consistent with the electroweak data, the LW scale must be of a few $\mathrm{TeV}[4,6-8]$. Currently, there is a vast literature both as phenomenological issues and as theoretical aspects of the LWSM [9-21], which shows the growing interest in the last few years.

Since LW quantum electrodynamics is the cornerstone of the LWSM, issues concerning its properties can give a valuable insight into our understanding of the LW dynamics. A fundamental feature regarding quantum field theories (QFT) is associated with ultraviolet divergences. This shortcoming is intrinsic to interacting QFTs when excitations are point particles and interactions are local, which arise due the fact that the integrals over intermediate energies diverge at their high energy end [22]. At the classical context, Podolsky [23], Podolsky and Kikuchi [24, 25], Montgomery [26], and Green $[27,28]$ developed completely relativistic electrodynamics which is free from the defect of infinities self-energies and which reduces to Maxwell-Lorentz formulation for low energy phenomenon through the addition of higher-order derivative kinetic terms in Maxwell electrodynamics. This field theory has the advantage of maintaining the $U(1)$ gauge structure of the QED. In the quantum realm, Sakurai gave 
an outlook relative to divergence difficulties inherent to quantum theory at high energies [29]. In order to obtain finite values of physical observables as mass and charge of the electron in QED, the photon propagator must be modified by a cut-off parameter, as Pauli-Villars regulator for example. But the Pauli-Villars prescription is unsatisfactory since it gives rise to non-Hermitian interactions and scattering processes do not conserve probabilities, violating thereby the unitarity. Sakurai suspected that QED must be modified at short distances to overcome these difficulties at fundamental level. Soon after Sakurai's insight, Lee and Wick proposed a way to treat the QED divergences in ultraviolet range $[1,2]$. The basic idea was to promote the Pauli-Villars regulator as a freedom degree in QED. The outcome is a modified photon propagator that in high frequencies limit goes as $k^{-4}$, improving the behavior of the electrodynamics at short distances. Their model is named Lee-Wick finite theory of QED. Nevertheless, a wrong sign residue appears in the poles, coming to light negative norm states in the Hilbert space and thereby breaking unitarity. Lee and Wick argued that these ghost modes should possess a heavy mass and decay in onshell particles. It is worth to note that Lee-Wick original ideas were abandoned only after the dimensional regularization schema of gauge theories [30], living a relegation era for about two decades. Nevertheless, questions in regard to the foundations of the Lee-Wick finite QED remain open until today; for example, demonstration concerning the unitarity at arbitrary loops in perturbative formalism does not exist; however, no exceptions were found and satisfactory answers are not yet settled.

Following up previous works [31, 32], the focus of this paper is on the electromagnetic sector of the LW quantum electrodynamics and it is organized as follows. In Section 2, we review some properties of the abelian Lee-Wick electrodynamics. In Section 3, the higher-order canonical quantization is considered in detail. In Section 4 , we estimate a quantum bound in the LW heavy mass using the experimental value of the anomalous magnetic moment of the electron. In Section 5, we discuss the possibility of coexistence of magnetic monopoles and the duality symmetry in the LW model. Our conclusions are presented in Section 6.

In our conventions $\hbar=c=1$ and the signature of the metric is $(+1,-1,-1,-1)$.

\section{Overview of the Abelian Lee-Wick Model}

The Abelian LW model is defined by the following gaugeinvariant Lagrangian:

$$
\mathscr{L}=-\frac{1}{4} F_{\mu \nu} F^{\mu \nu}-\frac{1}{4 M^{2}} F_{\mu \nu} \square F^{\mu \nu}
$$

where $F_{\mu \nu}\left(=\partial_{\mu} A_{\nu}-\partial_{\nu} A_{\mu}\right)$ is the field strength.

Let us then show that the above Lagrangian describes two independent (on-shell) spin-1 fields: massless one and massive one, with positive and negative norm, respectively. To do that, it is appropriate to provide another formulation where an auxiliary field is introduced and the higher-derivative term is absent. The field theory with real Vectorial fields $A_{\mu}$ and $Z_{\mu}$ with Lagrangian

$$
\begin{aligned}
\mathscr{L}= & \frac{1}{2} A_{\mu} \square Z^{\mu}+\frac{1}{2} \partial_{\mu} A^{\mu} \partial_{\nu} Z^{\nu}-\frac{M^{2}}{8} A_{\mu} A^{\mu} \\
& +\frac{M^{2}}{4} A_{\mu} Z^{\mu}-\frac{M^{2}}{8} Z_{\mu} Z^{\mu},
\end{aligned}
$$

is equivalent to the field theory with the Lagrangian in (1). In fact, varying $Z_{\mu}$ gives

$$
Z_{\mu}=A_{\mu}+\frac{2}{M^{2}} \square A_{\mu}-\frac{2}{M^{2}} \partial_{\mu} \partial_{\nu} A^{\nu}
$$

and the coupled second-order equations from (2) are fully equivalent to the fourth-order equations from (1). The system (2) now separates cleanly into the Lagrangians for two fields, when we make the change of variables

$$
\begin{aligned}
& A_{\mu}=B_{\mu}+C_{\mu}, \\
& Z_{\mu}=B_{\mu}-C_{\mu} .
\end{aligned}
$$

In terms of $B_{\mu}, C_{\mu}, B_{\mu \nu} \equiv \partial_{\mu} B_{\nu}-\partial_{\nu} B_{\mu}$, and $C_{\mu \nu} \equiv \partial_{\mu} C_{\nu}-$ $\partial_{\nu} C_{\mu}$, the Lagrangian now becomes

$$
\mathscr{L}=-\frac{1}{4} B_{\mu \nu} B^{\mu \nu}+\frac{1}{4} C_{\mu \nu} C^{\mu \nu}-\frac{M^{2}}{2} C_{\mu} C^{\mu},
$$

which is nothing but the difference of the Maxwell Lagrangian for $B_{\mu}$ and the Proca Lagrangian for $C_{\mu}$.

The particle content of the theory can also be obtained directly from (1). To accomplish this goal we compute the residues at the simple poles of the saturated propagator (contraction of the propagator with conserved currents). Adding to (1) the gauge-fixing term $\mathscr{L}_{\lambda}=-(1 / 2 \lambda)\left(\partial_{\mu} A^{\mu}\right)^{2}$, where as usual $\lambda$ plays the role of the gauge-fixing parameter, and noting that due to the structure of the theory and the choice of a linear gauge-fixing functional, no FaddeevPopov ghosts are required in this case, we promptly get the propagator in momentum space; namely,

$$
\begin{aligned}
D_{\mu \nu}(k)=\frac{M^{2}}{k^{2}\left(k^{2}-M^{2}\right)}\left\{\eta_{\mu \nu}\right. & -\frac{k_{\mu} k_{\nu}}{k^{2}} \\
& \left.\times\left[1+\lambda\left(\frac{k^{2}}{M^{2}}-1\right)\right]\right\} .
\end{aligned}
$$

Contracting (6) with conserved currents $J^{\mu}(k)$ yields

$$
\begin{aligned}
\mathscr{M} & \equiv J^{\mu} D_{\mu \nu} J^{\nu} \\
& =-\frac{J^{2}}{k^{2}}+\frac{J^{2}}{k^{2}-M^{2}},
\end{aligned}
$$

which allows us to conclude, taking into account that $J^{2}<0$ [33-35], that the signs of the residues of $\mathscr{M}$ at the poles $k^{2}=0$ and $k^{2}=M^{2}$ are, respectively,

$$
\operatorname{Res} \mathscr{M}\left(k^{2}=0\right)>0, \quad \operatorname{Res} \mathscr{M}\left(k^{2}=M^{2}\right)<0,
$$

which confirms with our previous result. 
It is worth noticing that the wrong sign of the residue of the heavy particle indicates the instability of the theory at the classical level. From the quantum point of view it means that the theory is nonunitary. Luckily, these difficulties can be circumvented. Indeed, the classical instability can be removed by imposing a future boundary condition in order to prevent exponential growth of certain modes. However, this procedure leads to causality violation in the theory [36]; fortunately, this acausality is suppressed below the scales associated with the LW particles. On the other hand, Lee and Wick argued that, despite the presence of the aforementioned degrees of freedom associated with a nonpositive definite norm on the Hilbert space, the theory could nonetheless be unitary as long as the new LW particles obtain decay widths. There is no general proof of unitary at arbitrary loop order for the LW electrodynamics; nevertheless, there is no known example of unitarity violation. Accordingly, the LW electrodynamics is finite. Therefore, we need not be afraid of the massive spin-1 ghost.

In summary, we may say that the LW work consists essentially in the introduction of Pauli-Villars, wrong-sign propagator, fields as physical degrees of freedom which leads to amplitudes that are better behaved in the ultraviolet and render the logarithmically divergent QED finite.

We remark that, for the sake of convenience, we will work on the representation of the gauge field $A_{\mu}$ as given in (1), with the propagator as in (6).

\section{Lee-Wick Canonical Quantization}

Higher-order canonical quantization has been performed for some authors in the past [37-40]. Nevertheless, some questionable results are presented in these works. We initially will accomplish the canonical quantization of the Lee-Wick electromagnetism and at the end of this section discuss these controversial outcomes. Our starting point for the higherorder canonical quantization is the following LW Lagrangian density:

$$
\mathscr{L}=-\frac{1}{4} F_{\mu \nu}^{2}+\frac{1}{2 M^{2}} \partial_{\mu} F_{\alpha \beta} \partial^{\mu} F^{\alpha \beta} .
$$

We will analyze (9) instead of (1) since these Lagrangians are the same up to a total derivate. This choice is due to the fact that the Lagrangian (1) has third-order derivative fields, which introduce additional complications in the canonical quantization. The pairs of canonically conjugate variables related to Lagrangian (9) are $\left(A^{\alpha}, \pi_{\alpha}\right)$ and $\left(\bar{A}^{\alpha}, \eta_{\alpha}\right)$, respectively, where $\bar{A}^{\alpha} \equiv \dot{A}^{\alpha}$ is an independent variable. Since gauge invariance holds in the LW model, the second-order Lagrangian (9) is degenerate; that is, the Hessian matrix is singular.

A set of generalized canonical momenta are

$$
\begin{gathered}
\pi^{\nu}=-F^{0 \nu}+\frac{1}{M^{2}}\left(\square F^{0 \nu}+\partial_{0} \partial_{i} F^{i \nu}\right), \\
\eta^{\nu}=\frac{1}{M^{2}} \partial_{0} F^{0 \nu} .
\end{gathered}
$$

Primary constraints are obtained from the definition of the canonical momenta, without making use of the equations of motion. According to relations (10), the LW model has the following primary constraints:

$$
\begin{gathered}
\eta_{0} \approx 0, \\
\pi_{0}+\partial_{i} \eta_{i} \approx 0,
\end{gathered}
$$

where " $\approx$ " means weak equations according to Dirac's method [41-44]. It is necessary to evaluate the dynamics of the constraints. As usual in Hamiltonian formalism, it is required to compute the canonical Hamiltonian, which is given by

$$
\begin{aligned}
H_{C}=\int d^{3} \mathbf{x}[ & \left.\pi^{\alpha} \bar{A}_{\alpha}+\eta^{\alpha} \dot{\bar{A}}-\mathscr{L}\right] \\
=\int d^{3} \mathbf{x}[ & \pi_{0} \bar{A}_{0}-\pi_{i} \bar{A}_{i}+\bar{A}_{0}\left(\partial_{i} \eta_{i}\right)-\frac{1}{2} M^{2} \eta_{i}^{2} \\
& +\left(\partial_{j} \eta_{i}\right) F_{j i}-\frac{1}{2} F_{0 i}^{2}+\frac{1}{4} F_{i j}^{2}-\frac{1}{2 M^{2}} \partial_{i} F_{i 0} \partial_{j} F_{j 0} \\
& \left.\quad-\frac{1}{4 M^{2}} \partial_{0} F_{i j} \partial_{0} F_{i j}+\frac{1}{4 M^{2}} \partial_{k} F_{i j} \partial_{k} F_{i j}\right] .
\end{aligned}
$$

The primary Hamiltonian $H_{1}$ is defined as

$$
H_{1}=H_{C}+\int d^{3} \mathbf{x}\left[\lambda_{1} \eta_{0}+\lambda_{2}\left(\pi_{0}+\partial_{i} \eta_{i}\right)\right] .
$$

So, applying the Poisson brackets we obtain

$$
\begin{gathered}
\dot{\eta}_{0}=\left\{\eta_{0}, H_{1}\right\} \approx 0, \\
\left(\dot{\pi}_{0}+\partial_{i} \dot{\eta}_{i}\right)=\left\{\pi_{0}+\partial_{i} \eta_{i}, H_{1}\right\}=\partial_{i} \pi^{i} \approx 0,
\end{gathered}
$$

which reveal the appearance of a nonprimary constraint to us. The secondary constraint arises from the condition that the primary constraints should be preserved in time. It is necessary to identify all the constraints of the model. Then, computing the Poisson brackets again, but now with the secondary Hamiltonian

$$
H_{2}=H_{C}+\int d^{3} \mathbf{x}\left[\lambda_{1} \eta_{0}+\lambda_{2}\left(\pi_{0}+\partial_{i} \eta_{i}\right)+\lambda_{3} \partial_{i} \pi_{i}\right],
$$

we get $\partial_{i} \dot{\pi}_{i}=\left\{\partial_{i} \pi_{i}, H_{2}\right\} \approx 0$. No more constraints appear in our formalism, which implies that the consistency condition is identically fulfilled.

Analyzing the Hamilton field equations of motion to $A_{0}$ and $A_{i}$ provides us with

$$
\begin{aligned}
& \dot{A}_{0}=\left\{A_{0}, H\right\}=\bar{A}_{0}+\lambda_{2}, \\
& \dot{A}_{i}=\left\{A_{i}, H\right\}=\bar{A}_{i}+\partial_{i} \lambda_{3},
\end{aligned}
$$

which allow us to choose the following Lagrange multipliers:

$$
\lambda_{2}=0, \quad \lambda_{3}=0 .
$$


On the other hand, the equations of motion concerning $\bar{A}_{i}$ and $\bar{A}_{0}$ yield

$$
\begin{gathered}
\dot{\bar{A}}_{i}=\left\{\bar{A}_{i}, H\right\}=M^{2} \eta_{i}+\partial_{i} \bar{A}_{0}, \\
\dot{\bar{A}}_{0}=\left\{\bar{A}_{0}, H\right\}=\lambda_{1} .
\end{gathered}
$$

Equation (18) is nothing but $\eta^{i}=\left(1 / M^{2}\right) \partial_{0} F^{0 i}$. The function related to the $\lambda_{1}$ is found through (19). The equations related to $\eta_{j}, \pi_{0}$, and $\pi_{j}$ do not introduce further information. The extended Hamiltonian can be expressed as $H_{E}=H_{C}+$ $\int d^{3} \mathbf{x} \dot{\bar{A}}_{0} \eta_{0}$, which gives us

$$
\begin{aligned}
H_{E}=\int d^{3} \mathbf{x}[ & \pi_{0} \bar{A}_{0}-\pi_{i} \bar{A}_{i}+\bar{A}_{0}\left(\partial_{i} \eta_{i}\right)-\frac{1}{2} M^{2} \eta_{i}^{2} \\
& +\left(\partial_{j} \eta_{i}\right) F_{j i}-\frac{1}{2} F_{0 i}^{2}+\frac{1}{4} F_{i j}^{2}-\frac{1}{2 M^{2}} \partial_{i} F_{i 0} \partial_{j} F_{j 0} \\
& \left.\quad-\frac{1}{4 M^{2}} \partial_{0} F_{i j} \partial_{0} F_{i j}+\frac{1}{4 M^{2}} \partial_{k} F_{i j} \partial_{k} F_{i j}+\dot{\bar{A}}_{0} \eta_{0}\right] .
\end{aligned}
$$

The constraints obtained are all of the first class; that is, the Poisson bracket with all the other constraints vanishes identically, which is a direct consequence of $U(1)$ gauge invariance. The canonical quantization requires that we impose a gauge choice and remove the nonphysical variables. The gauge condition necessary to change the set of the first class constraints into set of the second class constraints is obtained by analysing the LW field equations in terms of potential $A^{\mu}$, which can be expressed as

$$
\left(1+\frac{\square}{M^{2}}\right) \square A^{\nu}-\partial^{\nu}\left(1+\frac{\square}{M^{2}}\right) \partial_{\mu} A^{\mu}=0 .
$$

Equation (21) suggests that a possible gauge choice is

$$
\left(1+\frac{\square}{M^{2}}\right) \partial_{\mu} A^{\mu}=C,
$$

where $C$ is an arbitrary constant which can be chosen as to equal to zero, which provides us with the following gauge conditions:

$$
\bar{A}_{0}=0, \quad\left(1+\frac{\square}{M^{2}}\right) \nabla \cdot \mathbf{A}=0, \quad A_{0}=0 .
$$

The LW field equation (21) is compatible with this particular gauge choice. (We could also choose the gauge conditions $\bar{A}_{0}=0, \nabla \cdot \mathbf{A}=0, A_{0}=0$.) The gauge constraints also satisfy the consistency condition. All the constraints obtained are now of the second class type and are given by

$$
\begin{gathered}
\Omega_{1}=\eta_{0} \approx 0, \quad \Omega_{2}=\pi_{0}+\partial_{i} \eta_{i} \approx 0, \\
\Omega_{3}=\partial_{i} \pi_{i} \approx 0, \quad \Omega_{4}=\bar{A}_{0} \approx 0, \\
\Omega_{5}=\left(1+\frac{\square}{M^{2}}\right) \partial_{i} A_{i} \approx 0, \quad \Omega_{6}=A_{0} \approx 0 .
\end{gathered}
$$

A set of constraints found enable us to determine the constrained matrix. The only elements of the $C_{\alpha \beta} \equiv\left\{\Omega_{\alpha}, \Omega_{\beta}\right\}$ nonzero are

$$
\begin{gathered}
\left\{\Omega_{1}, \Omega_{4}\right\}=-\left\{\Omega_{4}, \Omega_{1}\right\}=\delta^{3}\left(\mathbf{x}-\mathbf{x}^{\prime}\right), \\
\left\{\Omega_{2}, \Omega_{6}\right\}=-\left\{\Omega_{6}, \Omega_{2}\right\}=\left(1-\frac{\nabla^{2}}{M^{2}}\right) \nabla^{2} \delta^{3}\left(\mathbf{x}-\mathbf{x}^{\prime}\right), \\
\left\{\Omega_{3}, \Omega_{5}\right\}=-\left\{\Omega_{5}, \Omega_{3}\right\}=\delta^{3}\left(\mathbf{x}-\mathbf{x}^{\prime}\right) .
\end{gathered}
$$

Since the constraints are all of the second class, the $C$ matrix is invertible and the Dirac brackets are completely characterized by

$$
\begin{aligned}
\left\{A(x), B\left(x^{\prime}\right)\right\}_{D} \\
=\left\{A(x), B\left(x^{\prime}\right)\right\} \\
\quad-\int d^{3} \mathbf{y} d^{3} \mathbf{z}\left\{A(x), \Omega_{a}(y)\right\} C_{a b}^{-1}(y, z)\left\{A(x), B\left(x^{\prime}\right)\right\} .
\end{aligned}
$$

Computing the inverse $C$-matrix and taking into account that the green function $G\left(\mathbf{x}-\mathbf{x}^{\prime}\right)$ satisfy the equation (1$\left.\nabla^{2} / M^{2}\right) \nabla^{2} G\left(\mathbf{x}-\mathbf{x}^{\prime}\right)=-\delta^{3}\left(\mathbf{x}-\mathbf{x}^{\prime}\right)$ and this is given by

$$
G\left(\mathbf{x}-\mathbf{x}^{\prime}\right)=\frac{1}{4 \pi} \frac{1}{\left|\mathbf{x}-\mathbf{x}^{\prime}\right|}\left[1-e^{-M\left|\mathbf{x}-\mathbf{x}^{\prime}\right|}\right],
$$

and then the Dirac brackets becomes

$$
\begin{aligned}
& \left\{A^{\mu}(\mathbf{x}, t), \pi^{\nu}\left(\mathbf{x}^{\prime}, t\right)\right\}_{D} \\
& =\left(\eta^{\mu \nu}-\eta^{\mu 0} \eta^{\nu 0}\right) \delta^{3}\left(\mathbf{x}-\mathbf{x}^{\prime}\right) \\
& \quad+\eta^{\mu i} \eta^{\nu j}\left(1-\frac{\nabla^{2}}{M^{2}}\right) \frac{\partial}{\partial x^{i}} \frac{\partial}{\partial x^{\prime j}} G\left(\mathbf{x}-\mathbf{x}^{\prime}\right), \\
& \left\{\bar{A}^{\mu}(\mathbf{x}, t), \eta^{\nu}\left(\mathbf{x}^{\prime}, t\right)\right\}_{D} \\
& \quad=\left(\eta^{\mu \nu}-\eta^{\mu 0} \eta^{\nu 0}\right) \delta^{3}\left(\mathbf{x}-\mathbf{x}^{\prime}\right) .
\end{aligned}
$$

According to Dirac's method, we must write these equations as strong equalities. Using the canonical quantization prescription $\left(\{A, B\}_{D} \rightarrow-i[A, B]\right)$, the LW commutators are given by

$$
\begin{gathered}
{\left[A_{i}(\mathbf{x}, t), \pi_{j}(\mathbf{x}, t)\right]} \\
=i \delta_{i j} \delta^{3}\left(\mathbf{x}-\mathbf{x}^{\prime}\right)+i\left(1-\frac{\nabla^{2}}{M^{2}}\right) \partial_{i} \partial_{j}^{\prime} G\left(\mathbf{x}-\mathbf{x}^{\prime}\right), \\
{\left[\bar{A}_{i}(\mathbf{x}, t), \eta_{j}\left(\mathbf{x}^{\prime}, t\right)\right]=i \delta_{i j} \delta^{3}\left(\mathbf{x}-\mathbf{x}^{\prime}\right) .}
\end{gathered}
$$

It is worth noting that, in the absence of higherorder derivative terms, the commutator (29) reproduces the Maxwell commutation relation. To end up, it is important to note that the Poincaré algebra is also satisfied. 
The history of the quantization of higher-order electromagnetic theories began a long time ago. The first who tried to perform the higher-order quantization in the electromagnetism were Podolsky and Kikuchi in 1944 [24] and some years later Podolsky and Schwed [45] using the Glupta-Bleuer method; the results obtained however are dubious due to the fact that in that epoch there was not an easy method to deal with quantization methods of gauge theories, a procedure that is not completely clear up to now. In the 1950s, Pitman Jr. [46] and Richard E. Martin [47] probed some aspects of Podolsky electrodynamics in their Ph.D. thesis. Again, the results found are not correct due the inability of treating the electron self-energy.

Higher-order canonical quantization by Dirac formalism began in the early 1960s. Since then, some authors tried to achieve the quantization of higher-order theories [48-51] via Dirac's method; on the other hand, canonical quantization of higher-order electromagnetism starting from the determination of primary and secondary constraints has been performed in [37-40]. Nevertheless, there are some misconceptions in previous works. In $[37,38]$, the density Lagrangian is incorrect. On the other hand, in [37], the number of primary constraints is 2, while in [38] the same theory discussed in [37] introduces only one primary constraint. As previously stated, primary constraints follow solely from the definition of the canonical momenta, while nonprimary constraints arise directly from the condition that the primary constraints hold in time [41-44]. In [37, 40] the authors argue that the gauge $\nabla \cdot \mathbf{A}=0$ cannot be used, but only $\left(1+\square / M^{2}\right) \nabla \cdot \mathbf{A}=0$ can be used. In [38], in turn, it is claimed that both gauges are feasible. Upon gauge fixation, a set of the first class constraints and gauge conditions turn into a second class constraints [41-44]. Any gauge condition can be used provided it is consistent with field equations. We hope with this brief discussion to close the misunderstanding concerning the higher-order canonical quantization. We still emphasize that the Lagrangian (9) used by us is not the same as the previous one the authors used. Nevertheless, the physical content is completely equivalent, as it should be.

\section{Anomalous Magnetic Moment of the Electron and the LW Heavy Mass}

Taking into account that QED predicts the anomalous magnetic moment of the electron correctly to ten decimal places, a quantum bound on the mass $M$ of the LW heavy particle can be found by computing the anomalous electron magnetic moment in the context of the LW electrodynamics and comparing afterwards the result obtained with that of QED. To accomplish this goal, we recall that the anomalous magnetic moment stems from the vertex correction for the scattering of the electron by an external field, as it is shown in Figure 1.

For an electron scattered by an external static magnetic field and in limit $\mathbf{q} \rightarrow \mathbf{0}$, the gyromagnetic ratio is given by [52]

$$
g=2\left[1+F_{2}(0)\right]
$$

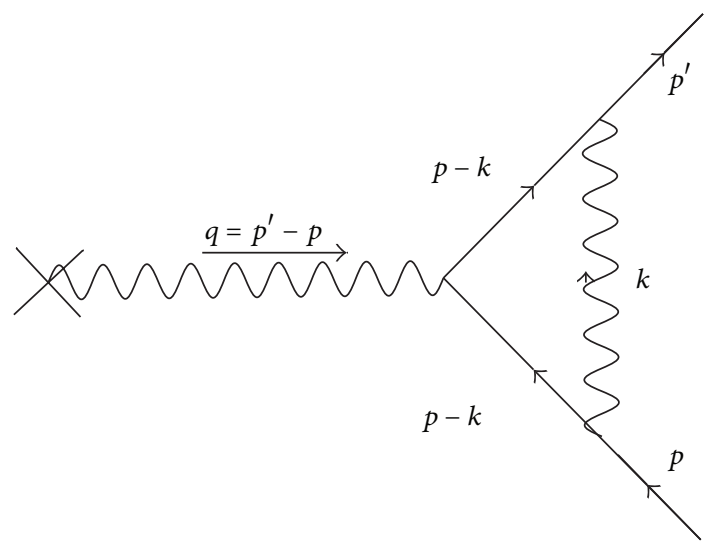

FIGURE 1: Vertex correction for electron scattering by an external field.

The form factor of the electron, $F_{2}(0)$, corresponds to a shift in the $g$-factor, usually quoted in the form $F_{2}(0) \equiv$ $(g-2) / 2$, and yields the anomalous magnetic moment of the electron. By employing (31) in the calculation of the diagram in Figure 1, it can be shown that

$$
\begin{aligned}
F_{2}(0)=\frac{\alpha}{\pi} \int_{0}^{\infty} & d \alpha_{1} d \alpha_{2} d \alpha_{3} \delta\left(1-\Sigma \alpha_{i}\right) \\
& \times\left[\frac{\alpha_{1}}{\alpha_{2}+\alpha_{3}}-\frac{\alpha_{1}^{2}\left(\alpha_{2}+\alpha_{3}\right)}{\left(\alpha_{2}+\alpha_{3}\right)^{2}+\alpha_{1} / \varepsilon}\right],
\end{aligned}
$$

where $\varepsilon \equiv m^{2} / M^{2}$, with $m$ being the electron mass. We call attention to the fact that the term $-M^{2} k^{\mu} k^{\nu} / k^{4}\left(k^{2}-M^{2}\right)[1+$ $\left.\lambda\left(k^{2} / M^{2}-1\right)\right]$ that appears in (6) makes no contribution to the the form factor $F_{2}(0)$ because the propagator always occurs coupled to conserved currents.

Integrating the above expression first with respect to $\alpha_{3}$ and subsequently with respect to $\alpha_{2}$ gives

$$
\begin{aligned}
F_{2}(0) & =\frac{\alpha}{\pi} \int_{0}^{1} d \alpha_{1} \int_{0}^{1-\alpha_{1}} d \alpha_{2}\left[\frac{\alpha_{1}}{1-\alpha_{1}}-\frac{\alpha_{1}\left(1-\alpha_{1}\right)}{\left(1-\alpha_{1}\right)^{2}+\alpha_{1} / \varepsilon}\right] \\
& =\frac{\alpha}{\pi} \int_{0}^{1} d \alpha_{1} \frac{\alpha_{1}^{2}}{\alpha_{1}+\varepsilon\left(1-\alpha_{1}\right)^{2}} .
\end{aligned}
$$

Computing $F_{2}(0)$, we arrive at the conclusion that

$$
\begin{aligned}
F_{2}(0) \approx \frac{\alpha}{2 \pi}[ & 1-\frac{2}{3}\left(\frac{m}{M}\right)^{2}-2\left(\frac{25}{12}+\ln \left(\frac{m}{M}\right)\right)\left(\frac{m}{M}\right)^{4} \\
& \left.+\mathcal{O}\left(\left(\frac{m}{M}\right)^{6}\right)\right] .
\end{aligned}
$$

The first term of the above equation is equal to that calculated by Schwinger in 1948 [53]. Since then $F_{2}(0)$ has been calculated to order $\alpha^{10}$ for QED. The second term of (34) is the most important correction related to the parameter $M$ of the LW electrodynamics. 
Recent calculations concerning $F_{2}(0)$ in the framework of QED give for the electron [54]

$$
F_{2}(0)=1159652181.78(0.06)(0.04)(0.02) \times 10^{-12},
$$

where the uncertainty comes mostly from that of the best non-QED value of the fine structure constant $\alpha$. The current experimental value for the anomalous magnetic moment is, in turn, [55]

$$
F_{2}(0)=1159652180.73(0.28) \times 10^{-12} .
$$

Comparing the theoretical value predicted by QED with the experimental one shows that these results agree in 1 part in $10^{12}$. As a consequence,

$$
\frac{2}{3}\left(\frac{m}{M}\right)^{2}<10^{-12}
$$

Consequently, a lower limit on the heavy particle Lee and Wick hypothesized that the existence is $M \approx 409 \mathrm{GeV}$.

\section{Duality Symmetry and Monopoles in the Lee-Wick Electrodynamics}

An important question concerning the LW finite QED is whether or not the LW heavy particle and magnetic charge can live in peace in its context. To answer this question we introduce magnetic current $J_{m}^{\mu}=\left(\rho_{m}, \mathbf{J}_{m}\right)$ in the LW dual field equations. It is fairly straightforward to show that the resulting system of modified higher-order field equations, namely,

$$
\begin{gathered}
\left(1+\frac{\square}{M^{2}}\right) \partial_{\mu} F^{\mu \nu}=J_{e}^{v}, \\
\partial_{\mu} \widetilde{F}^{\mu \nu}=J_{m}^{\nu}
\end{gathered}
$$

where $\widetilde{F}^{\mu \nu}=(1 / 2) \epsilon^{\mu \nu \alpha \beta} F_{\alpha \beta}\left(\epsilon^{0123}=+1\right)$, describes the existence of a magnetic charge. In fact, assuming the absence of electric fields, charges, and currents and the absence of magnetic current, we are left essentially with two equations for the magnetic field which have the familiar Dirac monopole solution $\mathbf{B}=\left(q_{m} / 4 \pi r^{2}\right) \widehat{\mathbf{r}}$, where $q_{m}$ is the magnetic charge. Using the usual methods, the Dirac quantization condition $\left(q_{e} q_{m} / 4 \pi\right)=n / 2$, where $q_{e}$ is the electric charge and $n$ is an integer, can be promptly recovered. We have thus succeeded in finding a consistent system of Maxwell + Vectorial boson mass + magnetic charge equations. We remark that the Dirac monopole and the massive Vectorial boson cannot coexist in the context of Proca massive electrodynamics [56] because the latter, unlike the LW QED, is not gauge invariant. The very existence of the Dirac monopole is undoubtedly linked to the existence of the gauge invariance of the corresponding theory. Interestingly enough, the system formed by (38) and (39) is not symmetric under the duality transformation $F^{\mu \nu} \rightarrow \widetilde{F}^{\mu \nu}, \widetilde{F}^{\mu \nu} \rightarrow-F^{\mu \nu}$, augmented by $J_{e}^{\mu} \rightarrow J_{m}^{\mu}$, $J_{m}^{\mu} \rightarrow-J_{e}^{\mu}$. This fact raises an interesting question: would it be possible to accommodate simultaneously magnetic charge and duality transformations in the framework of a higher-order electromagnetic model? A good attempt in this direction might be, for instance, the model defined by the field equations

$$
\begin{aligned}
& \left(1+\frac{\square}{M^{2}}\right) \partial_{\mu} F^{\mu \nu}=J_{e}^{v}, \\
& \left(1+\frac{\square}{M^{2}}\right) \partial_{\mu} \widetilde{F}^{\mu \nu}=J_{m}^{\nu}
\end{aligned}
$$

since it is symmetric under duality transformations. It is worth noticing that $\left(1+\square / M^{2}\right) \partial_{\mu} \widetilde{F}^{\mu \nu}=J_{m}^{\nu}$ is identically zero in the absence of the magnetic current. Let us see then whether this model admits a monopole-like solution. For a magnetostatic charge of strength $q_{m}$ fixed at the origin, the solution of the preceding equations is

$$
\mathbf{B}=\frac{q_{m}}{4 \pi}\left[\frac{1-(1+M r) e^{-M r}}{r^{2}}\right] \widehat{\mathbf{r}},
$$

which for large distances reduces to the Dirac result, as it should be. Our point, nonetheless, is to ascertain whether or not this solution describes a magnetic monopole at short distances. To see this we calculate the flux of the radial magnetic field through a spherical surface $\partial \mathscr{R}$ of radius $r$ with the static monopole of strength $q_{m}$ at its center. Performing the computation we promptly find $\oint_{\partial \mathscr{R}} \mathbf{B} \cdot \widehat{\mathbf{n}} d A=q_{m}[1-(1+$ $\left.M r) e^{-M r}\right]$ which implies that, for $M r \ll 1, \oint_{\partial \mathscr{R}} \mathbf{B} \cdot \widehat{\mathbf{n}} d A \approx$ 0 . Now, taking into account that if $\mathbf{B}=\nabla \times \mathbf{A}, \oint_{\partial \mathscr{R}} \mathbf{B}$. $\widehat{\mathbf{n}} d A$ vanishes identically, we come to conclusion that $\mathbf{A}$ can exist everywhere in the region under consideration, which shows us that in the short range limit the Dirac quantization condition cannot be recovered. To see if this actually occurs, we take into account that, for $M r \ll 1, \mathbf{B} \approx\left(q_{m} M^{2} / 8 \pi\right) \widehat{\mathbf{r}}$, implying that the magnetic field is constant at short distances instead of falls down with $1 / r^{2}$. This bizarre behavior of the magnetic field certainly precludes us from recovering the Dirac quantization condition. One heuristic way of seeing that is to consider the motion of a particle of mass $m$ and charge $q_{e}$ in the field of the magnetic monopole. From the equation of motion of the electric charge, $m \ddot{\mathbf{r}}=q_{e} \dot{\mathbf{r}} \times \mathbf{B}$, we get the ratio of change of its angular momentum

$$
\frac{d}{d t}(\mathbf{r} \times m \dot{\mathbf{r}})=\frac{q_{e} q_{m} M^{2} r^{2}}{8 \pi} \frac{d \widehat{\mathbf{r}}}{d t},
$$

a result that prevents us from defining a conserved total angular momentum as in the case of the Dirac monopole. Now, if the distances are neither too large nor much small, the potential vector cannot exist everywhere in the domain bounded by $\partial \mathscr{R}$ because $F^{\mu \nu}$ satisfies (41) rather than (39). Unlucky, we could not overcome this difficult by introducing the concept of a string as Dirac did since in this case $\nabla$. $\mathbf{B}=\left(q_{m} M^{2} / 4 \pi\right)\left(e^{-M r} / r\right)$ does not vanishe anywhere in the aforementioned domain. The preceding analysis leads us to conjecture that Dirac-like monopoles and duality transformations cannot be accommodated in the context of one and same higher-order electromagnetic model. 


\section{Final Remarks}

In this paper, we have studied many issues of the electromagnetic sector of the LW model. First, we saw that questions related to higher-order canonical quantization can be very tricky, as for instance, the definition of primary and nonprimary constraints as well as the gauge conditions. Extensive argumentation why it is the correct way to proceed in higher-order canonical quantization was accomplished at the end of Section 3.

About magnetic monopoles, a possible road of investigation concerning Lorentz violation theories was carried out. One interesting theory to evaluate this scenario is the Myers-Pospelov (MP) model. MP is an effective higher-order gauge invariant theory that violates the Lorentz symmetry in the electromagnetic sector. It would be interesting to analyze the eventual existence of magnetic monopoles and the presence of dual symmetry in a model that violates the Lorentz symmetry together with higher-order derivative terms. Another field of great interest would be the search for monopoles in the non-abelian generalization of Lee-Wick QED. In the context of LWSM, the search for monopoles can be achieved by finding topologically nontrivial finite-energy solutions.

To conclude, the bound we have found on the LW heavy mass was obtained using the most accurate experimental data currently available as input for the anomalous magnetic moment of the electron. As far as the truly (loop) quantum effects are concerned, a quick glance at (37) clearly shows that a better agreement between theory and experiment concerning the anomalous magnetic moment of the electron would lead to the improvement of the quantum bound. Consequently, there is great probability of setting a better quantum bound on the LW heavy mass in the foreseeable future.

\section{Conflict of Interests}

The authors declare that there is no conflict of interests regarding the publication of this paper.

\section{Acknowledgments}

Rodrigo Turcati is very grateful to $\mathrm{CNPq}$ (a Brazilian agency) for providing the financial support. The authors are very grateful to the referees for their helpful suggestions and comments.

\section{References}

[1] T. D. Lee and G. C. Wick, "Negative metric and the unitarity of the S-matrix," Nuclear Physics B, vol. 9, no. 2, pp. 209-243, 1969.

[2] T. D. Lee and G. C. Wick, "Finite theory of quantum electrodynamics," Physical Review D, vol. 2, pp. 1033-1048, 1970.

[3] B. Grinstein, D. O'Connell, and M. Wise, “The Lee-Wick standard model," Physical Review D, vol. 77, Article ID 025012, 2008 .
[4] E. Alvarez, C. Schat, L. Da Rold, and A. Szynkman, "Electroweak precision constraints on the Lee-Wick standard model," Journal of High Energy Physics, vol. 2008, no. 04, p. 026, 2008.

[5] F. Wu, M. Zhong, and D. Phys. Rev, “TeV scale Lee-Wick fields out of large extra dimensional gravity," Physical Review D, vol. 78, Article ID 085010, 2008.

[6] C. D. Carone and R. F. Lebed, "Minimal Lee-Wick extension of the Standard Model," Physics Letters B, vol. 668, no. 3, pp. 221225, 2008.

[7] T. E. J. Underwood and R. Zwicky, "Electroweak precision data and the lee-wick standard model," Physical Review D, vol. 79, Article ID 035016, 2009.

[8] R. S. Chivukula, A. Farzinnia, R. Foadi, and E. H. Simmons, "Custodial isospin violation in the Lee-Wick standard model," Physical Review D, vol. 81, no. 9, Article ID 095015, 2010.

[9] B. Fornal, B. Grinstein, and M. B. Wise, "Lee-Wick theories at high temperature," Physics Letters B, vol. 674, no. 4-5, pp. 330335, 2009.

[10] B. Grinstein, D. O'Connell, and M. B. Wise, "Massive vector scattering in Lee-Wick gauge theory," Physical Review D, vol. 77, Article ID 065010, 2008.

[11] J. R. Espinosa, B. Grinstein, D. O'Connell, and M. B. Wise, "Neutrino masses in the Lee-Wick standard model," Physical Review D, vol. 77, Article ID 085002, 2008.

[12] B. Grinstein and D. O'Connell, "One-loop renormalization of Lee-Wick gauge theory," Physical Review D, vol. 78, Article ID 105005, 2008.

[13] J. R. Espinosa and B. Grinstein, "Ultraviolet properties of the Higgs sector in the Lee-Wick standard model," Physical Review D, vol. 83, no. 7, Article ID 075019, 2011.

[14] C. D. Carone and R. F. Lebed, "A higher-derivative Lee-Wick standard model," Journal of High Energy Physics, vol. 2009, no. 1 , article 43, 2009.

[15] C. D. Carone, "Higher-derivative Lee-Wick unification," Physics Letters B, vol. 677, pp. 306-310, 2009.

[16] A. Rodigast and T. Schuster, "No Lee-Wick fields out of gravity," Physical Review D, vol. 79, Article ID 125017, 2009.

[17] Y.-F. Cai, T.-T. Qiu, R. Brandenberger, and X.-M. Zhang, "Nonsingular cosmology with a scale-invariant spectrum of cosmological perturbations from Lee-Wick theory," Physical Review D, vol. 80, Article ID 023511, 2009.

[18] T. G. Rizzo, "Searching for Lee-Wick gauge bosons at the LHC," Journal of High Energy Physics, vol. 2007, no. 6, article 70, 2007.

[19] T. G. Rizzo, "Unique identification of Lee-Wick gauge bosons at linear colliders," Journal of High Energy Physics, vol. 1, article 042, 2008.

[20] T. R. Dulaney and M. B. Wise, "Flavor changing neutral currents in the Lee-Wick Standard Model," Physics Letters B, vol. 658, no. 5, pp. 230-235, 2008.

[21] F. S. Gama, M. Gomes, J. R. Nascimento, A. Y. Petrov, and A. J. da Silva, "Higher-derivative supersymmetric gauge theory," Physical Review D, vol. 84, no. 4, Article ID 045001, 2011.

[22] R. Jackiw, "What good are quantum field theory infinities?" in Mathematical Physics 2000, A. Fokas, A. Grigoryan, T. Kibble, and B. Zegarlinski, Eds., p. 326, Imperial College Press, London, UK, 2000.

[23] B. Podolsky, "A generalized electrodynamics part I-nonquantum," Physical Review, vol. 62, p. 68, 1942.

[24] B. Podolsky and C. Kikuchi, "A Generalized Electrodynamics Part II-Quantum," Physical Review, vol. 65, pp. 228-235, 1944. 
[25] B. Podolsky and C. Kikuchi, "Auxiliary conditions and electrostatic interaction in generalized quantum electrodynamics," vol. 67, pp. 184-192, 1945.

[26] D. J. Montgomery, "Relativistic interaction of electrons on Podolsky's generalized electrodynamics," Physical Review, vol. 69, pp. 117-124, 1946.

[27] A. E. Green, "Self-energy and interaction energy in Podolsky's generalized electrodynamics," Physical Review, vol. 72, pp. 628631, 1947.

[28] E. S. Green, "On infinites in generalized meson-field theory," Physical Review B, vol. 73, pp. 26-29, 1948.

[29] J. J. Sakurai, Advanced Quantum Mechanics, 1967.

[30] G. 't Hooft and M. Veltman, "Regularization and renormalization of gauge fields," Nuclear Physics Section B, vol. 44, no. 1, pp. 189-213, 1972.

[31] A. Accioly and E. Scatena, "Limits on the coupling constant of higher-derivative electromagnetism," Modern Physics Letters A, vol. 25, no. 4, pp. 269-276, 2010.

[32] A. Accioly, P. Gaete, J. Helayël-Neto, E. Scatena, and R. Turcati, "Investigations in the Lee-Wick electrodynamics," Modern Physics Letters A, vol. 26, no. 26, pp. 1985-1994, 2011.

[33] A. Accioly, "Quadratic gravity theories in $2+1$ dimensions and the topological Chern-Simons term," Physical Review D, vol. 67, no. 12, Article ID 127502, 2003.

[34] A. Accioly, "Can one cure the nonunitarity of three-dimensional quadratic gravity using a topological Chern-Simons term?" Nuclear Physics B: Proceedings Supplement, vol. 127, pp. 100-104, 2004.

[35] A. Accioly and M. Dias, "Algorithm for probing the unitarity of topologically massive models," International Journal of Theoretical Physics, vol. 44, no. 8, pp. 1123-1139, 2005.

[36] S. Coleman, "Ettore Majorana school on subnuclear phenomena," in Proceedings of Erice 1969, p. 282, Academic Press, New York, NY, USA, 1970.

[37] C. A. Galvão and B. M. Pimentel, "The canonical structure of Podolsky generalized electrodynamics," Canadian Journal of Physics. Revue Canadienne de Physique, vol. 66, no. 5, pp. 460466, 1988.

[38] J. Barcelos-Neto, C. Galvão, and C. Natividade, "Quantization of Podolsky theory in the BFV formalism," Zeitschrift für Physik C, vol. 52, no. 4, pp. 559-562, 1991.

[39] A. Dutra and M. Hott, "Quantization of maxwell-chernsimons-podolsky theory," http://arxiv.org/abs/hep-th/9403167.

[40] R. Bufalo, B. M. Pimentel, and G. E. R. Zambrano, "Path integral quantization of generalized quantum electrodynamics," Physical Review D: Particles, Fields, Gravitation and Cosmology, vol. 83, no. 4, Article ID 045007, 2011.

[41] P. A. M. Dirac, "Generalized Hamiltonian dynamics," Canadian Journal of Mathematics, vol. 2, pp. 129-148, 1950.

[42] P. A. M. Dirac, Lectures on Quantum Mechanics, Belfer Graduate School of Science, Yeshiva University, New York, NY, USA, 1964.

[43] A. Hanson, T. Regge, and C. Teitelboim, Constrained Hamiltonian Systems, Academic Nazionale dei Lincei, Rome, Italy, 1976.

[44] K. Sundermeyer, Constrained Dynamics, Lectures Notes in Physics, Springer, Berlin, Germany, 1982.

[45] B. Podolsky and P. Schwed, "Review of a generalized electrodynamics," Reviews of Modern Physics, vol. 20, pp. 40-50, 1948.

[46] G. R. Pitman Jr., University of Cincinnati, 1955.

[47] Martin and R. Eugene, Electron self energy in generalized quantum electrodynamics [Ph. D. Dissertation], University of Cincinnati, 1960.
[48] J. Barcelos-Neto and N. Braga, "Higher order canonical formalism in field theory and constraints," Acta Physica Polonica B, vol. 20, p. 205, 1989.

[49] J. Barcelos-Neto and N. R. Braga, "Some quantum aspects of the regularization with higher derivatives," Modern Physics Letters A, vol. 4, no. 22, pp. 2195-2200, 1989.

[50] J. Barcelos-Neto and C. P. Natividade, "Hamiltonian path integral formalism with higher derivatives," Zeitschrift für Physik C: Particles and Fields, vol. 51, no. 2, pp. 313-319, 1991.

[51] S. I. Muslin, "Quantization of singular systems with second order Lagrangians," http://arxiv.org/abs/math-ph/0010020vl.

[52] P. H. Frampton, Gauge Field Theories, vol. 62 of Frontiers in Physics, Benjamin/Cummings, 1987.

[53] J. Schwinger, "On quantum-electrodynamics and the magnetic moment of the electron," Physical Review, vol. 73, no. 4, pp. 416417, 1948.

[54] T. Aoyama, M. Hayakawa, T. Kinoshita, and M. Nio, "Complete tenth-order QED contribution to the Muon g-2," Physical Review Letters, vol. 109, Article ID 111807, 2012.

[55] D. Hanneke, S. Fogwell, and G. Gabrielse, "New measurement of the electron magnetic moment and the fine structure constant," Physical Review Letters, vol. 100, no. 12, Article ID 120801, 2008.

[56] A. Y. Ignatiev and G. C. Joshi, "Massive electrodynamics and the magnetic monopoles," Physical Review D, vol. 53, no. 2, pp. 984-992, 1996. 

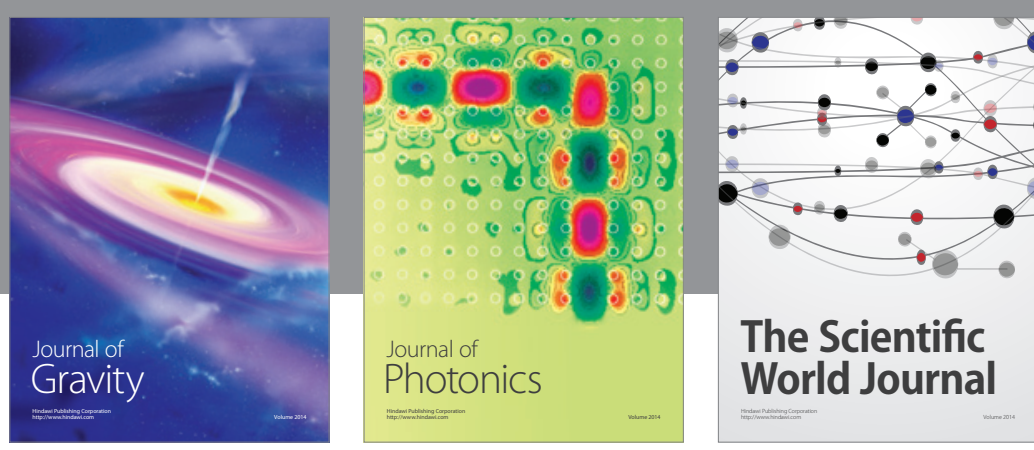

The Scientific World Journal
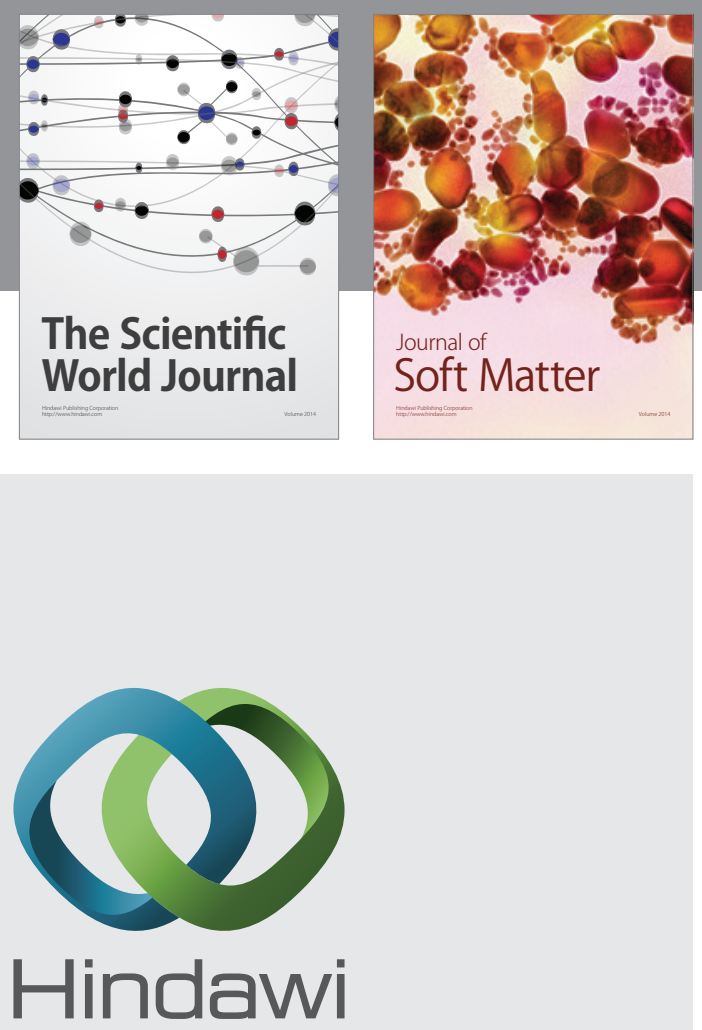

Submit your manuscripts at

http://www.hindawi.com

nternational Journal of

Statistical Mechanics
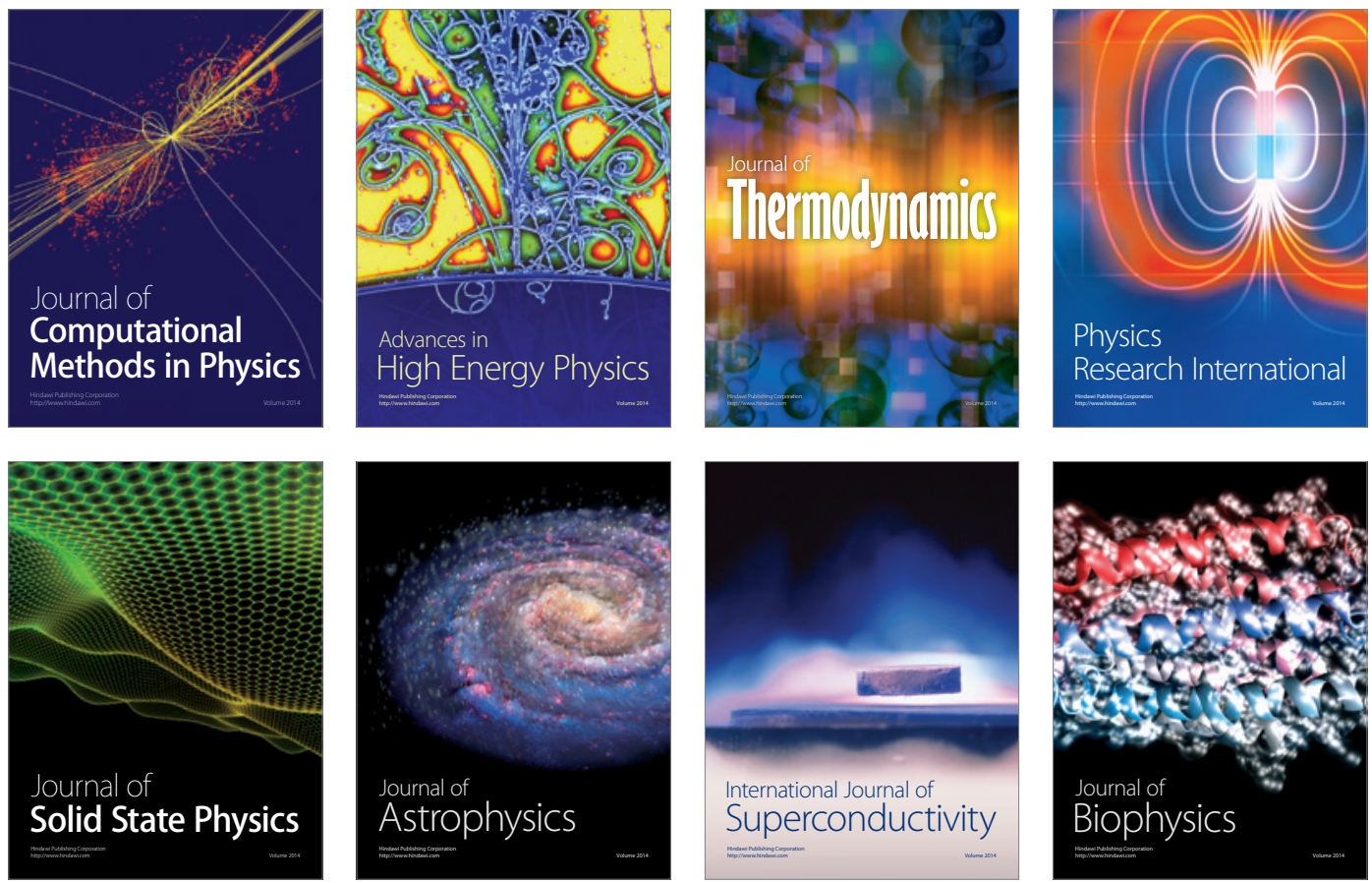
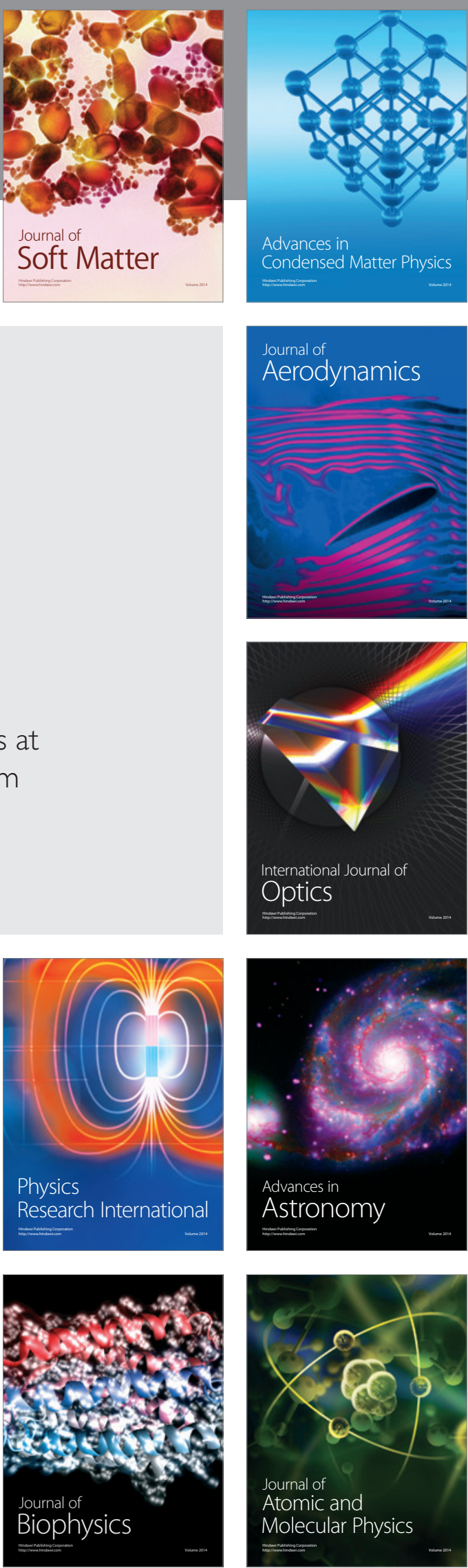